\title{
SKATEBOARD: A HUMAN CONTROLLED NON-HOLONOMIC SYSTEM
}

\author{
Balazs Varszegi \\ Department of Applied Mechanics \\ Budapest University of Technology \\ and Economics \\ Budapest, Hungary $\mathrm{H}-1111$ \\ Email: varszegi@mm.bme.hu
}

\author{
Denes Takacs \\ MTA-BME Research Group on \\ Dynamics of Machines and Vehicles \\ Budapest, Hungary, $\mathrm{H}-1111$ \\ takacs@mm.bme.hu
}

\author{
Gabor Stepan \\ Department of Applied Mechanics \\ Budapest University of Technology \\ and Economics \\ Budapest, Hungary, $\mathrm{H}-1111$ \\ stepan@mm.bme.hu
}

\begin{abstract}
A simple mechanical model of the skateboard-skater system is analyzed, in which a linear PD controller with delay is included to mimic the effect of human control. The equations of motion of the non-holonomic system are derived with the help of the Gibbs-Appell method. The linear stability analysis of rectilinear motion is carried out analytically using the D-subdivision method. It is shown how the control gains have to be varied with respect to the speed of the skateboard in order to stabilize the uniform motion. The critical reflex delay of the skater is determined as a function of the speed and the fore-aft location of the skater on the board. Based on these, an explanation is given for the well-known instability of the skateboard-skater system at high speed.
\end{abstract}

\section{INTRODUCTION}

Skateboard was invented only in 1950's and already enjoys worldwide popularity. The challenge of understanding the motion of the skateboard is due to the kinematic constraints and the complexity of the skater-skateboard system. If the skateboard is tilted around its longitudinal axis then the wheel-pairs also turn around their steering axes because of the wheel mounting geometry. This phenomenon makes the mechanical model non-holonomic and the equation of motion can be derived by means of the Gibbs-Appell method [1]. The effect of speed on the stability of the skateboard was investigated a not long after the invention of skateboards [2] but publications (see, for exam- ple, [3]) appear even nowadays. The speed dependent stability of non-holonomic systems still have unexplored behaviors although their dynamics have been investigated for more than 100 years. This is clearly shown by recent publications, where the lateral stability of the bicycle and the three-dimensional biped walking machines are studied (see, for example, $[4,5]$ ).

Another interesting challenge is to explain the balancing effort of the person on the skateboard. Reflex delay in the control loop usually plays an important role in dynamical systems, as is shown by papers on the human balancing problem from biological to engineering points of view [6-8].

In this paper, a mechanical model of the skateboard is constructed in which human control is taken into account. We can say, that the skater wants to control his angle by a torque at his ankle, what is produced by muscles. This is an usual approach in modeling of human control, when we model the human as a robot, what is able to act with forces and torques $[6,8]$.

So we use a very simple linear PD control loop to apply torque at the skaters ankle, and we also consider the reflex delay of human control. The stability analysis of the rectilinear motion is investigated analytically, and a case study is presented to emphasize the physical meaning of the results. Let us note here, other control models can better represent the higher-frequency behavior (for example McRuer approach [9]). This phenomenon can be investigated in further researches after this paper. 


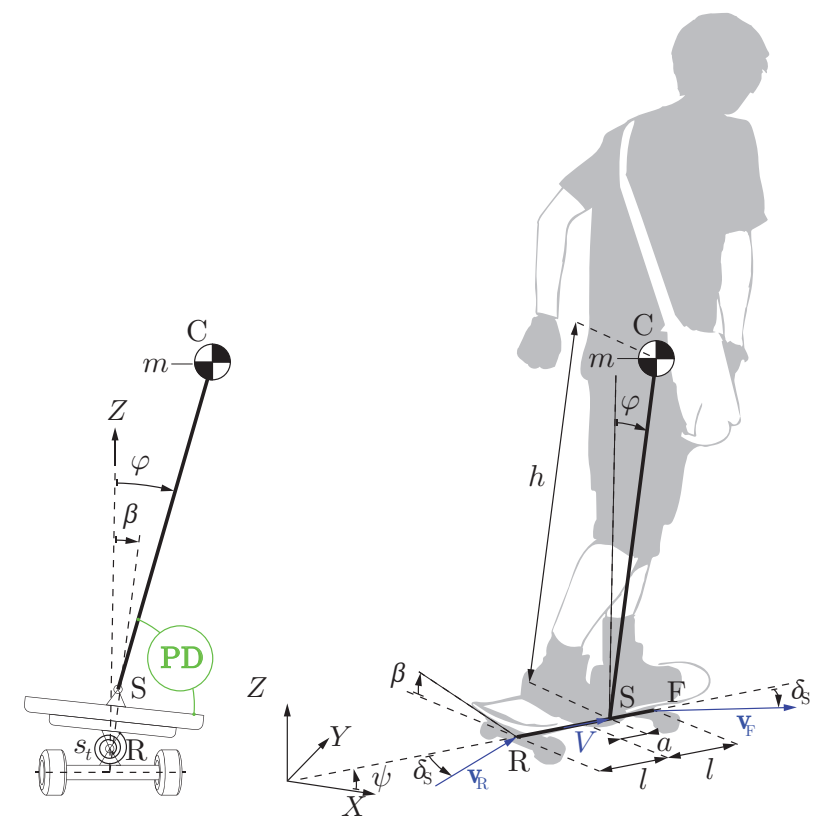

FIGURE 1. THE MECHANICAL MODEL OF THE SKATEBOARD-SKATER SYSTEM

\section{THE MECHANICAL MODEL}

The mechanical model in question (see Fig. 1) is based on [3] and [10], where simpler mechanical models of the skateboard were used but a human control-loop was also implemented. Here, a more realistic model is constructed, in which the board and the skater can move separately and a control torque acts at the skater's ankle.

The model consists of two massless rods. One of them models the skateboard between the front $(\mathrm{F})$ and rear $(\mathrm{R})$ points. Although we neglect the mass of the board its mass moment of inertia around its longitudinal axis is taken into account. The other rod between the center point $(\mathrm{S})$ of the contact patch between the skater and the board and the mass center (C) models the skater having a lumped mass. These two rods are connected by a hinge parallel to the longitudinal axis at the point $S$. The geometrical parameters are the following: $2 h$ denotes the height of the skater, $2 l$ is the length of the board, $a$ characterizes the location of the skater on the board ( $a>0$ means that the skater stands in front of the center of the board), the mass point $m$ represents the mass of the skater, $J_{\mathrm{b}}$ is the mass moment of inertia of the skateboard around its longitudinal axis and $g$ stands for of the gravity acceleration $\left(g=9.81\left[\mathrm{~m} / \mathrm{s}^{2}\right]\right)$.

In this study, we do not consider the loss of contact between the wheels and the ground, consequently that the longitudinal axis of the skateboard is always parallel to the ground. One can choose five generalized coordinates to describe the motion: $X$ and $Y$ are the coordinates of the point $\mathrm{S} ; \psi$ describes the longitu- dinal direction of the skateboard; $\varphi$ is the deflection angle of the skater from the vertical direction; and finally, $\beta$ is the deflection angle of the board (see Fig. 1).

As it is also shown in Fig. 1, the rotation of the skateboard about its longitudinal axis is resisted by the torsional spring of stiffness $s_{\mathrm{t}}$, which is originated in the special wheel suspension system of the skateboard. The rotation of the skater around the longitudinal axis of the skateboard is opposed by the internal torque of the PD controller. Hereby, the rectilinear motion is considered as the desired motion, which means that zero torque is produced by the controller when the deflection angle of the skater is zero (i.e.: $\varphi=0$ ). Thus, the control torque can be calculated as

$$
M_{\mathrm{PD}}(t)=P \varphi(t-\tau)+D \dot{\varphi}(t-\tau)
$$

where $\tau$ refers to the time delay, $P$ and $D$ represent the proportional and the differential control gains, respectively.

Regarding the rolling wheels of the skateboard, kinematic constraining equations can be given for the velocities $\mathbf{v}_{\mathrm{F}}, \mathbf{v}_{\mathrm{R}}$ of points $\mathrm{F}$ and $\mathrm{R}$. The directions of the velocities of these points depend on the deflection angle $\beta$ of the board through the socalled steering angle $\delta_{\mathrm{S}}$ (see Fig. 1). This connection can be described by the expression:

$$
\sin \beta \tan \kappa=\tan \delta_{\mathrm{S}},
$$

where $\kappa$ is the fixed complementary angle of the so-called rake angle in the skateboard wheel suspension system $[3,10]$. Based on this, two scalar kinematic constraining equations can be constructed:

$$
\begin{gathered}
(-\sin \psi+\cos \psi \sin \beta \tan \kappa) \dot{X}+ \\
(\cos \psi+\sin \psi \sin \beta \tan \kappa) \dot{Y}+(l-a) \dot{\psi}=0, \\
(\sin \psi+\cos \psi \sin \beta \tan \kappa) \dot{X}+ \\
(-\cos \psi+\sin \psi \sin \beta \tan \kappa) \dot{Y}+(l+a) \dot{\psi}=0 .
\end{gathered}
$$

We also introduce a third kinematic constraint, namely, the longitudinal speed $V$ of the board is kept constant:

$$
\dot{X} \cos \psi+\dot{Y} \cos \psi=V
$$

We do not present details, but it can be proven that the condition of the linear stability of rectilinear motion of skateboard remains the same even if the constraint Eqn. (5) does not hold, nevertheless, it makes our calculations simpler. Since all of the kinematic constraints (Eqns. (3)-(5)) are linear combinations of the generalized velocities they can be written in the following form:

$$
\mathbf{A}(\mathbf{q}) \cdot \dot{\mathbf{q}}=\mathbf{A}_{0}
$$


where

$$
\begin{aligned}
& \mathbf{q}^{\mathrm{T}}=\left[\begin{array}{lllll}
X & Y & \psi & \phi & \beta
\end{array}\right],
\end{aligned}
$$

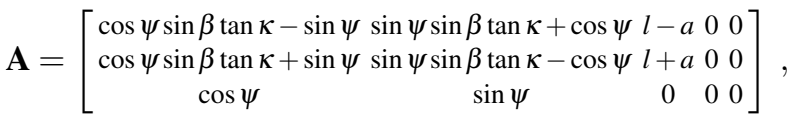

$$
\begin{aligned}
& \mathbf{A}_{0}^{\mathrm{T}}=\left[\begin{array}{lll}
0 & 0 & V
\end{array}\right] .
\end{aligned}
$$

Note, that if the mass moment of inertia $J_{\mathrm{b}}$ of the board is zero, then $\beta$ and $\varphi$ are not independent and $\beta$ can be expressed as a function of $\varphi$ and $\dot{\varphi}$. Thus, the kinematic constraints are not linear functions of the generalized velocities if $J_{\mathrm{b}}=0$, and the Gibbs-Appell method can not be used. Therefore, we consider the case when $J_{\mathrm{b}}>0$.

In order to apply the Gibbs-Appell equation (see in [1]), pseudo velocities have to be chosen, by which the kinematic constraints can be eliminated. In our case, two pseudo velocities are required since the difference of the numbers of the generalized coordinates and the kinematic constraints is two. An appropriate choice can be the angular velocity components of the skater and the skateboard around the longitudinal axis, respectively:

$$
\sigma_{1}(t):=\dot{\varphi}(t) \quad \text { and } \quad \sigma_{2}(t):=\dot{\beta}(t)
$$

The generalized velocities can be expressed with the help of these two pseudo velocities and with the generalized coordinates:

$$
\left[\begin{array}{c}
\dot{X} \\
\dot{Y} \\
\dot{\psi} \\
\dot{\varphi} \\
\dot{\beta}
\end{array}\right]=\left[\begin{array}{c}
V\left(\cos \psi+\frac{a}{l} \tan \kappa \sin \beta \sin \psi\right) \\
V\left(\sin \psi-\frac{a}{l} \tan \kappa \sin \beta \cos \psi\right) \\
-\frac{V}{l} \tan \kappa \sin \beta \\
\sigma_{1} \\
\sigma_{2}
\end{array}\right]
$$

The derivation of both sides of this expression with respect to time leads to the generalized accelerations as functions of the pseudo accelerations, pseudo velocities and generalized coordinates.

During the derivation of the equation of motion, the socalled energy of acceleration $(\mathscr{A})$ is needed. For a rigid body it can be computed as:

$\mathscr{A}=\frac{1}{2} m \mathbf{a}_{\mathrm{G}} \cdot \mathbf{a}_{\mathrm{G}}+\frac{1}{2} \alpha^{\mathrm{T}} \mathbf{J}_{\mathrm{G}} \alpha+\alpha^{\mathrm{T}}\left(\omega \times\left(\mathbf{J}_{\mathrm{G}} \omega\right)\right)+\frac{1}{2} \omega^{T}\left(\mathbf{J}_{\mathrm{G}} \omega\right) \omega^{2}$, where $\mathrm{G}$ refers to the center of the gravity of the body and $\mathbf{a}_{\mathrm{G}}$ is the acceleration of $\mathrm{G}$. The angular acceleration and the angular velocity are denoted by $\alpha$ and $\omega$, respectively. The mass of the body and its matrix of mass moment of inertia are $m$ and $\mathbf{J}_{\mathrm{G}}$, respectively. In case of skateboarding, there are two rigid bodies: the skater with a lumped mass $m$ at $\mathrm{C}$ and the board with mass moment of inertia $J_{\mathrm{b}}$ around the hinge axis, while the mass of the board is neglected compared to the mass of the skater. So the energy of acceleration forms:

$$
\begin{aligned}
& \mathscr{A}=\frac{m h V}{l^{2}} \tan \kappa \cos \varphi V \sin \beta(l-h \tan \kappa \sin \beta \sin \varphi) \dot{\sigma}_{1}+ \\
& \frac{m h a V}{l} \tan \kappa \cos \varphi \cos \beta \sigma_{2} \dot{\sigma}_{1}+\frac{m h^{2}}{2} \dot{\sigma}_{1}^{2}+\frac{1}{2} J_{\mathrm{b}} \cos ^{2}(2 \psi) \dot{\sigma}_{2}^{2}+\ldots
\end{aligned}
$$

The expressions that do not contain pseudo accelerations is not computed, because the equation of motion can be obtained in the form:

$$
\frac{\partial \mathscr{A}}{\partial \dot{\sigma}_{i}}=\Gamma_{i},
$$

where the right hand side is the pseudo force $\Gamma_{i}$ related to the $i$ th pseudo velocity $\sigma_{i}$. It can be determined from the virtual power of the active forces namely: the gravitational force, the torque produced by the controller and the torque produced by the spring:

$$
\delta P=m g h \sin \varphi \delta \sigma_{1}-s_{\mathrm{t}} \beta \delta \sigma_{2}+M_{\mathrm{PD}}(t)\left(\delta \sigma_{2}-\delta \sigma_{1}\right)
$$

where notation $\delta$ refers to the virtual quantities.

Thus the equations of motion are:

$$
\begin{aligned}
\dot{\sigma}_{1} & =\sin \varphi\left(\frac{g}{h}+\frac{V^{2}}{l^{2}} \tan ^{2} \kappa \sin ^{2} \beta \cos \varphi\right)-\frac{1}{h} M_{\mathrm{PD}}(t)+ \\
& -\frac{a}{h} \frac{V}{l} \tan \kappa \cos \beta \cos \varphi \sigma_{2}-\frac{V^{2}}{h l} \tan \kappa \sin \beta \cos \varphi \\
\dot{\sigma}_{2} & =\frac{M_{\mathrm{PD}}(t)-s_{\mathrm{t}} \sinh \beta}{\cos ^{2}(2 \psi) J_{\mathrm{b}}} \\
\dot{X} & =V\left(\cos \psi+\frac{a}{l} \tan \kappa \sin \beta \sin \psi\right), \\
\dot{Y} & =V\left(\sin \psi-\frac{a}{l} \tan \kappa \sin \beta \cos \psi\right), \\
\dot{\psi} & =-\frac{V}{l} \tan \kappa \sin \beta \\
\dot{\varphi} & =\sigma_{1} \\
\dot{\beta} & =\sigma_{2} .
\end{aligned}
$$

Note that $X, Y$ and $\psi$ are cyclic coordinates, so only the first two and the last two equations of Eqn. (16) have to be used for our further investigation.

\section{LINEAR STABILITY ANALYSIS}

In this section we are going to investigate the linear stability of the rectilinear motion of a skateboard-skater system. First, we take the linearized equation of motion around the stationary 
solution with respect to small perturbations in $\sigma_{1}, \sigma_{2}, \varphi$ and $\beta$. It can be written as

$$
\dot{\mathbf{X}}(t)=\mathbf{J} \cdot \mathbf{X}(t)+\mathbf{T} \cdot \mathbf{X}(t-\tau)
$$

where

$$
\begin{gathered}
\mathbf{J}=\left[\begin{array}{cccc}
0-\frac{a V}{h l} \tan \kappa & \frac{g}{h}-\frac{V^{2}}{h l} \tan \kappa \\
0 & 0 & 0 & -\frac{s_{\mathrm{t}}}{J_{\mathrm{b}}} \\
1 & 0 & 0 & 0 \\
0 & 1 & 0 & 0
\end{array}\right], \quad \mathbf{X}(t)=\left[\begin{array}{c}
\sigma_{1}(t) \\
\sigma_{2}(t) \\
\varphi(t) \\
\psi(t)
\end{array}\right], \\
\text { and } \quad \mathbf{T}=\left[\begin{array}{cccc}
-\frac{1}{m h^{2}} D & 0 & -\frac{1}{m h^{2}} P & 0 \\
\frac{1}{J_{\mathrm{b}}} D & 0 & \frac{1}{J_{\mathrm{b}}} P & 0 \\
0 & 0 & 0 & 0 \\
0 & 0 & 0 & 0
\end{array}\right]
\end{gathered}
$$

The characteristic function $D_{\mathrm{c}}(\lambda)$ can be determined by means of the substitution of the trial solution $\mathbf{X}(t)=\mathbf{K} \mathrm{e}^{\lambda t}(\mathbf{K} \in$ $\mathbb{C}^{4}$ and $\lambda \in \mathbb{C}$ ) into Eqn. (17). It leads to:

$$
\begin{aligned}
D_{\mathrm{c}}(\lambda) & =\frac{V \mathrm{e}^{-\lambda \tau}}{H J_{\mathrm{b}} l} \tan \kappa \mathrm{e}^{-\lambda \tau}(a \lambda+V)(D \lambda+P)+ \\
& +\frac{\mathrm{e}^{-\lambda \tau}}{m h^{2}}\left(\lambda^{2}+\frac{s_{\mathrm{t}}}{J_{\mathrm{b}}}\right)\left(D \lambda+m h e^{\lambda \tau}\left(h \lambda^{2}-g\right)+P\right)
\end{aligned}
$$

and the characteristic equation can be written in the form $D_{\mathrm{c}}(\lambda)=0$.

This equation has infinitely many complex roots. The fixed point in question, i.e. the rectilinear motion, is asymptotically stable if and only if all of the characteristic roots are situated in the left-half of the complex plane. The limit of stability corresponds to the case when the characteristic roots are located at the imaginary axis for some particular system parameters.

Two different types of stability boundaries can be distinguished; saddle-node (SN) bifurcation when both the real and imaginary parts of the characteristic root are zero, and Hopf bifurcation when the characteristic roots are pure complex. In our model, SN bifurcation occurs if $D_{\mathrm{c}}(0)=0$, namely:

$$
-\frac{g s_{\mathrm{t}}}{J_{\mathrm{b}} H}+\left(\frac{s_{\mathrm{t}}}{m J_{\mathrm{b}} H^{2}}+\frac{V^{2} \tan \kappa}{J_{\mathrm{b}} h l}\right) P=0 .
$$

The critical proportional gain $P_{\mathrm{SN}}$ can be determined:

$$
P_{\mathrm{SN}}=\frac{m g h l s_{\mathrm{t}}}{m H V^{2} \tan \kappa+s_{\mathrm{t}} l} .
$$

In case of Hopf bifurcation, the critical characteristic exponent can be written as pure imaginary numbers $\left(\lambda_{\mathrm{H}}= \pm \mathbf{i} \omega\right.$,
TABLE 1. PARAMETERS OF THE SKATER-BOARD SYSTEM

\begin{tabular}{|c|c|c|c|}
\hline$h[\mathrm{~m}]$ & $m[\mathrm{~kg}]$ & $a[\mathrm{~m}]$ & $\tau[\mathrm{s}]$ \\
\hline 0.85 & 75 & 0.05 & 0.24 \\
\hline \hline$s_{\mathrm{t}}[\mathrm{Nm} /$ rand $]$ & $l[\mathrm{~m}]$ & $\kappa\left[^{\mathrm{o}}\right]$ & $J_{\mathrm{b}}\left[\mathrm{kgm}^{2}\right]$ \\
\hline 100 & 0.3937 & 63 & $6.642 \cdot 10^{-3}$ \\
\hline
\end{tabular}
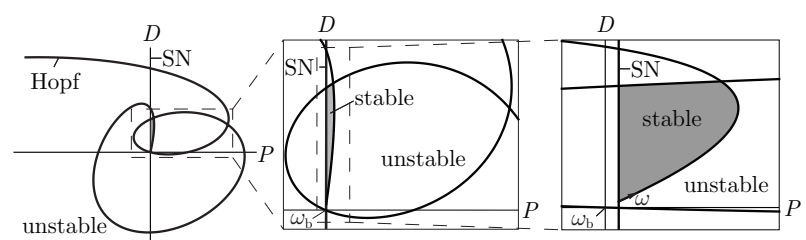

FIGURE 2. STRUCTURE OF STABILITY CHART

$\left.\omega \in \mathbb{R}^{+}\right)$. The characteristic equation $D_{\mathrm{c}}(\mathbf{i} \omega)$ can be separated into real and imaginary parts, which procedure is referred to Dsubdivision method. The critical control gains can be expressed:

$$
\begin{aligned}
P_{\mathrm{H}} & =\frac{p_{1}(\omega) p_{2}(\omega)}{p_{4}(\omega)}, \\
D_{\mathrm{H}} & =\frac{p_{1}(\omega) p_{3}(\omega)}{\omega p_{4}(\omega)},
\end{aligned}
$$

where

$$
\begin{aligned}
p_{1}(\omega) & =2 h l m \cos \kappa\left(g+h \omega^{2}\right)\left(J_{\mathrm{b}} \omega^{2}-s_{\mathrm{t}}\right) \\
p_{2}(\omega) & =l \cos \kappa\left(J_{\mathrm{b}} \omega^{2}-s_{\mathrm{t}}\right) \cos (\tau \omega)-m h V \sin \kappa(a \omega \sin (\tau \omega)+V \cos (\tau \omega)), \\
p_{3}(\omega) & =l \cos \kappa\left(J_{\mathrm{b}} \omega^{2}-s_{\mathrm{t}}\right) \sin (\tau \omega)+m h V \sin \kappa(a \omega \cos (\tau \omega)-V \sin (\tau \omega)), \\
p_{4}(\omega) & =\cos (2 \kappa)\left(l^{2}\left(s_{\mathrm{t}}-J_{\mathrm{b}} \omega^{2}\right)^{2}-h^{2} m^{2} V^{2}\left(a^{2} \omega^{2}+V^{2}\right)\right)+l^{2}\left(s_{\mathrm{t}}-J_{\mathrm{b}} \omega^{2}\right)^{2}+ \\
& +m^{2} h^{2} V^{2}\left(a^{2} \omega^{2}+V^{2}\right)+2 m h l V^{2} \sin (2 \kappa)\left(s_{\mathrm{t}}-J_{\mathrm{b}} \omega^{2}\right)
\end{aligned}
$$

Let us construct the linear stability chart of the rectilinear motion in the $P-D$ parameter plane (see in Fig. 2) for the realistic parameters of Tab. 1. The stability boundary given by Eqn. (22) starts like in case of the PD controlled inverted pendulum [6], but it does not spiral outwards continuously. Actually it will cross the origin of the parameter plane at $\omega=\omega_{\mathrm{b}}$, where

$$
\omega_{\mathrm{b}}=\sqrt{\frac{s_{\mathrm{t}}}{J_{\mathrm{b}}}}
$$

is the natural angular frequency of the skateboard without control (i.e. $P=0$ and $D=0$ corresponds to the switched off control). As a consequence, a loop of the stability boundary is showing up like for the simplified model in [10]). It can be proved, that only the internal region of this loop can be stable. As a summary, there are three different boundaries of the stable parameter domain: 


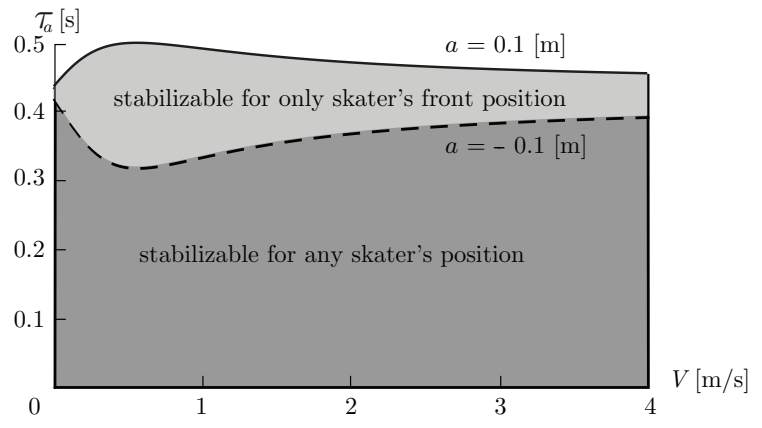

FIGURE 3. THE EFFECT OF THE SPEED ON THE ULTIMATE REFLEX TIME

the SN for static loss of stability and a low-frequency and a highfrequency Hopf bifurcation.

One can verify, that the above described closed loop of Hopf boundaries rotates counterclockwise around the origin as the reflex delay $\tau$ increases.

\section{EFFECTS OF THE LONGITUDINAL SPEED AND THE REFLEX DELAY}

When the effects of a human controller are neglected it can be shown that the higher the speed, the easier it is to stabilize the skateboard [2,3]. However, a more complex picture arises when the human control is taken into consideration. This was recognized in [10] using a simpler mechanical model that also exhibits rotating closed loop stability boundaries in the $P-D$ parameter plane.

Here we determine the necessary and sufficient conditions to stabilize the skateboard with respect to the skater's reaction time. A condition can be developed by examining the starting point of the D-curve. If the curve starts to the left hand side at $\omega=0$ then stable domain can not exist. This leads to the ultimate critical time delay $\tau_{\mathrm{cr}, \mathrm{u}}$ of the controller. If the delay is larger than this value, then the investigated equilibrium is unstable for sure. Fig. 3 shows the variation of the ultimate time delay versus the longitudinal speed for two different cases. The continuous line corresponds to $a=0.1 \mathrm{~m}$, i.e. the skater stands ahead of the center of the skateboard, while the dashed line corresponds to $a=-0.1 \mathrm{~m}$, i.e. the skater stands behind the center of the board. As it can be observed in the figure, the maximal time delay, by which the system can be stabilized, is larger for any speed when the skater stands in front of the center point of the board. However, in case of smaller reflex delay, the stabilization of the rectilinear motion is possible even when the skater stands behind the center of the board.

This can also be confirmed by practical experiences. Previous models without control-loop do not explain such behaviors of the skateboard. For example, it is proved in $[2,3]$ that the

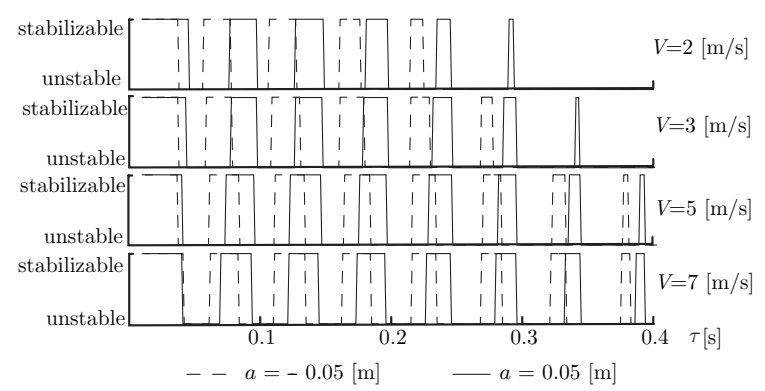

FIGURE 4. THE EFFECT OF THE REFLEX TIME ON THE STABILITY

rectilinear motion cannot be stable for any speed in case $a<0$.

Figure 3 also represents that the skater's position is more relevant at low speeds but both curves of the ultimate critical delays tend to the same value as the speed tends to infinity. Independently from the skater's position, the critical time delay is also the same at zero speed. The curves of Fig. 3 are characterized by

$$
\tau_{\mathrm{ut}, \max }^{V \rightarrow 0}=\sqrt{2} \sqrt{\frac{1}{\omega_{\mathrm{s}}^{2}}} \quad \text { and } \quad \tau_{\mathrm{ut}, \max }^{V \rightarrow \infty}=\sqrt{2} \sqrt{\frac{1}{\omega_{\mathrm{s}}^{2}}-\frac{1}{\omega_{\mathrm{b}}^{2}}}
$$

where

$$
\omega_{\mathrm{s}}=\sqrt{\frac{g}{h}} .
$$

The ultimate reflex time at zero speed $\left(\tau_{\mathrm{ut}, \max }^{V \rightarrow 0}\right)$ is identical with the critical time delay of the human balancing problem [6]. Based on the formulas, it is easy to show that $\tau_{\mathrm{ut}, \max }^{V \rightarrow 0}>\tau_{\mathrm{ut}, \max }^{V \rightarrow \infty}$ but the difference is very small for realistic parameters. For example, the parameters of Tab. 1 leads to $0.04 \%$ relative difference due to the fact that the natural angular frequency $\omega_{\mathrm{b}}$ is very high. The ultimate critical time (see in Fig. 3) is close, but higher, to the human reflex delay for hand [7].

As it was mentioned, former studies [3] proved that the system is unstable for $a<0$. Here we show that this statement can be reconsidered in some special cases, moreover, standing behind the center of the board can be even advantageous. In order to do this, the effect of the reflex time has to be investigated in more details. In Fig. 4, the existence of the stable $P-D$ parameter domain is illustrated versus the reflex delay. The figures are constructed for different longitudinal speeds. The continuous and the dashed lines belong to $a=0.05 \mathrm{~m}$ and $a=-0.05 \mathrm{~m}$, respectively. It can be observed that the condition $\tau<\tau_{\mathrm{ut} \text {,max }}$ is not sufficient from point of view of the stability. There are several reflex time ranges, where the system is not stabilizable for any control gains, which is due to the rotation of the Hopf loop around the origin 


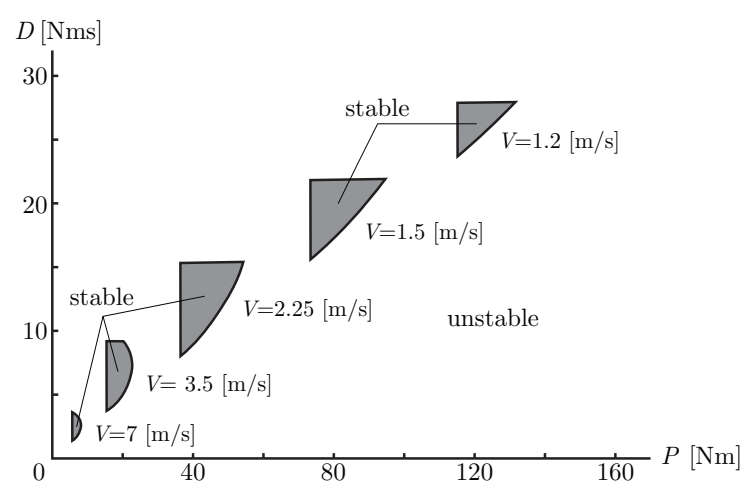

FIGURE 5. STABLE PARAMETER DOMAIN FOR DIFFERENT LONGITUDINAL SPEEDS

with increasing $\tau$. However, the locations of these ranges depend on the standing positions, namely, in some cases $a>0$, in other cases $a<0$ can lead to stable rectilinear motion. Nevertheless, if the speed tends to infinity, the applicable time delay ranges do not depend on the skater's position anymore.

Having a time delay below the sharp critical value is not enough to ensure stable rectilinear motion, the choice of adequate control gains $(P$ and $D)$ is also important. The variation of the stable domains in the $P-D$ parameter plane can be seen in Fig. 5 for $\tau=0.24 \mathrm{~s}$ and for different speeds.

The stable domain tends to the origin $(P=0, D=0)$ as the speed increases. As a consequence, the skater has to tune the control parameters more and more precisely in order to catch the very narrower and narrower stable parameter domain at increasing high speeds. Nevertheless, the switching off of the control (i.e., $P=0$ and $D=0$ ) could be a solution but only at infinite (very high) speed.

\section{CONCLUSION}

A mechanical model of the skateboard-skater system was constructed, in which the effect of the human balancing was taken into account by a linear delayed PD controller. The reflex time of the skater was also considered. The stability of the rectilinear motion of the skateboard was analyzed and stability charts were composed with special attention to the effects of the reflex time and the longitudinal speed.

Time delays were determined for realistic parameters, by which the skateboarding can be performed. The effect of the skater's position on the board was also investigated. It was verified, that the variation of the skater's position can qualitatively influence the stability of the rectilinear motion.

The presented stability charts can also explain the loss of stability at high speed. The stable parameter domain of the control gains reduces and its location tends towards the origin as the speed increases. As a consequence, the skater must decrease the control gains, which can enlarge the effects of the dead-zones of the human control. Clearly, the skater cannot apply close to zero control torque and cannot even sense very small tilting angles of the board. In human balancing models, the existences of dead-zones are also suspected as the reason of micro-chaotic and transient-chaotic vibrations around linearly unstable equilibrium with large surviving times [8].

\section{ACKNOWLEDGMENT}

This research was partly supported by the Janos Bolyai Research Scholarship of the Hungarian Academy of Sciences and by the Hungarian National Science Foundation under grant no. OTKA PD105442.

\section{REFERENCES}

[1] Gantmacher, F., 1975. Lectures in Analytical Mechanics. MIR Publisher, Moscow, Russia.

[2] Hubbard, M., 1980. "Human control of the skateboard". Journal of Biomechanics, 13, pp. 745-754.

[3] Kremnev, A. V., and Kuleshov, A. S., 2008. "Dynamics and simulation of the simplest model of skateboard". In Proceedings of Sixth EUROMECH Nonlinear Dynamics Conference.

[4] Kooijman, J. D. G., Meijaard, J. P., Papadopoulos, J. M., Ruina, A., and Schwab, A. L., 2011. "A bicycle can be self-stable without gyroscopic or caster effects". Science, 332, pp. 339-342.

[5] Wisse, M., and A, S., 2005. "Skateboard, bicycles, and three-dimensional biped walking machines: Velocitydependent stability by means of lean-to-yaw coupling". The International Journal of Robotics Research, 24(6), pp. 417-429.

[6] Stepan, G., 2009. "Delay effects in the human sensory system during balancing". Philosophical Transactions of The Royal Society, 367, pp. 1195-1212.

[7] Milton, J., Solodkin, A., Hlustik, P., and Small, S. L., 2007. "The mind of expert motor performance is cool and focused". NeuroImage, 35, pp. 804-813.

[8] Insperger, T., and Milton, J., 2014. "Sensory uncertainty and stick balancing at the fingertip". Biological Cybernetics, 108, pp. 85-101.

[9] McRuer, D. T., Weir, D. H., Jex, H. R., Magdeleno, R. E., and Allen, E. W., 1975. "Measurement of driver-vehicle multiloop response properties with a single disturbance input”. IEEE Transactions on systems, Man, and Cybernetics, SMC-5, pp. 490-497.

[10] Varszegi, B., Takacs, D., Stepan, G., and Hogan, S. J., 2014. "Balancing of the skateboard with reflex delay". In Proceedings of Eighth EUROMECH Nonlinear Dynamics Conference, ENOC2014. 